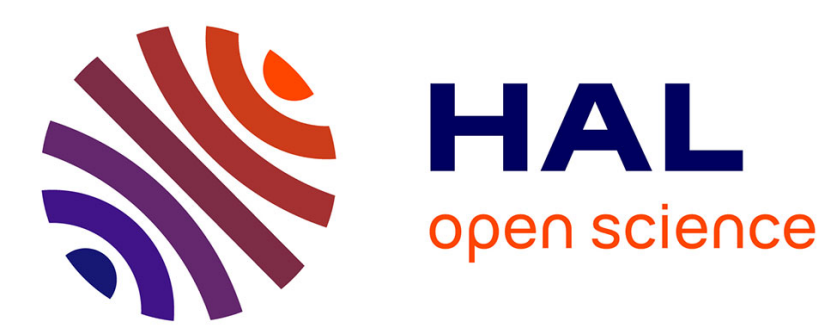

\title{
La naissance d'une commémoration de masse
}

Stéphane Michonneau

\section{To cite this version:}

Stéphane Michonneau. La naissance d'une commémoration de masse: Le cas barcelonais au début du XXe siècle. Sociétés \& Représentations, 2002, 13 (1), 10.3917/sr.013.0315 . hal-01674104

\section{HAL Id: hal-01674104 https://hal.science/hal-01674104}

Submitted on 1 Jan 2018

HAL is a multi-disciplinary open access archive for the deposit and dissemination of scientific research documents, whether they are published or not. The documents may come from teaching and research institutions in France or abroad, or from public or private research centers.
L'archive ouverte pluridisciplinaire HAL, est destinée au dépôt et à la diffusion de documents scientifiques de niveau recherche, publiés ou non, émanant des établissements d'enseignement et de recherche français ou étrangers, des laboratoires publics ou privés. 


\section{LA NAISSANCE D'UNE COMMÉMORATION DE MASSE}

Le cas barcelonais au début du xxe siècle

Stéphane Michonneau

\section{Publications de la Sorbonne | Sociétés \& Représentations}

$2002 / 1-n^{\circ} 13$

pages 315 à 331

ISSN 1262-2966

Article disponible en ligne à l'adresse:

http://www.cairn.info/revue-societes-et-representations-2002-1-page-315.htm

Pour citer cet article :

Michonneau Stéphane, “La naissance d'une commémoration de masse » Le cas barcelonais au début du xxe siècle, Sociétés \& Représentations, 2002/1 n 13, p. 315-331. DOI : 10.3917/sr.013.0315

Distribution électronique Cairn.info pour Publications de la Sorbonne.

(C) Publications de la Sorbonne. Tous droits réservés pour tous pays.

La reproduction ou représentation de cet article, notamment par photocopie, n'est autorisée que dans les limites des conditions générales d'utilisation du site ou, le cas échéant, des conditions générales de la licence souscrite par votre établissement. Toute autre reproduction ou représentation, en tout ou partie, sous quelque forme et de quelque manière que ce soit, est interdite sauf accord préalable et écrit de l'éditeur, en dehors des cas prévus par la législation en vigueur en France. II est précisé que son stockage dans une base de données est également interdit. 
HORS CADRE 


\section{LA NAISSANCE D'UNE COMMÉMORATION DE MASSE:}

\section{LE CAS BARCELONAIS AU DÉBUT DU XXe SIÈCLE}

\section{Stéphane Michonneau}

À la fin du XIXe siècle à Barcelone, un groupe étroit de promoteurs de mémoire peuple les comités exécutifs, les jurys artistiques, les commissions monumentales, les pétitions et les souscriptions. En monopolisant la gestion des souvenirs au nom de la collectivité qu'ils sont censés représenter, ces promoteurs non seulement définissent ce qui vaut ou pas d'être rappelé mais organise l'exercice pratique de la mémoire par le biais des commémorations publiques. De là s'organise une "société de mémoire " que l'on définira succinctement comme cette partie de la société catalane intéressée dans la détermination d'un passé commun.

Cette société commémorante relève d'un principe représentatif où le promoteur s'érige - et il est reconnu comme tel -, en expert des questions relatives au passé. En effet, la mémoire n'est pas l'affaire de tous: proposer une commémoration, ériger une statue demande une compétence particulière, une parole d'autorité prêtée quà peu de gens. Le modèle de l'expertise repose donc sur un processus de sélection sociale drastique afin que le comité ou la commission réunisse les meilleurs représentants possibles. Autrement dit, la logique de l'expert de mémoire est celle de l'élitisme: c'est pourquoi au XIXe siècle, les comités monumentaux rassemblent l'aréopage de la bonne société barcelonaise ${ }^{1}$.

Mais le modèle de l'expert change dans les années 1890, lorsque le modernisme remet en question les fondements de la domination sociale d'une frange étroite de la bourgeoisie commerçante, catholique et conservatrice. La professionnalisation des intellectuels n'est pas non plus étrangère à ce phénomène: pour un historien universitaire, un critique d'art ou un directeur de musée, la participation aux comités commémoratifs n'est plus aussi nécessaire pour asseoir son prestige et son autorité. Enfin, l'administration municipale gère de plus en plus ouvertement le souvenir de la cité et se dote des instruments bureaucratiques correspondants: commission monumentale propre, service du

1. Stéphane Michonneau, "Société et commémoration à Barcelone à la mi-XIXe siècle ", Genèse, n 40, sept. 2000, pp. 6-32. 
plan de la ville, architectes et urbanistes en chef, commission de réforme de la nomenclature. Ce faisant, l'administration est juge et partie: elle tranche plus souvent les conflits de mémoire dans la société commémorative et elle pèse de tout son poids administratif dans les décisions.

La crise des années 1890 prépare l'avènement des masses dans la société commémorative. L'événement est brutal car il correspond en Espagne à une crise politique majeure en 1898 lorsque le pays perd ses dernières colonies antillaises et asiatiques dans la guerre contre les États-Unis. En fait, le «Désastre » indique clairement l'épuisement du système politique de la Restauration monarchique comme régime autoritaire et antidémocratique dans ses principes. En Catalogne, l'irruption des masses dans la vie politique est contemporaine d'un mouvement nationaliste, le catalanisme, qui vise à refonder l'Espagne sur un modèle fédératif et à la convertir aux raisons du développement industriel. La mobilisation politique de fond se traduit à partir de 1901 par l'apparition de nouveaux partis de masse qui balaient aisément le vieux système politique en place: nationalistes et républicains inaugurent à Barcelone l'ère des masses qui ne triomphera dans le reste de l'Espagne qu'avec l'avènement de la II République, en 1931. Le cas catalan du début du XXe siècle revêt donc une importance capitale, au-delà de son localisme.

\section{La pétition: \\ une contestation populaire organisée}

Les nouvelles formes de la mobilisation collective sont multiples: l'étude de la société de mémoire est un prisme original qui permet d'en saisir l'essentiel. Un seul indice suffirait tout d'abord à indiquer sa massification. À la fin du XIXe siècle, les commissions monumentales présentent des listes de promoteurs, personnes dont la seule évocation atteste du sérieux, de l'importance du projet. En 1882 pour la première fois, c'est la fonction qui prime sur l'identité du représentant: on parle des présidents de l'Institut du Travail National, de l'Association artistico-archéologique, de l'École des Ingénieurs Industriels, de l'École d'architecture, etc. À partir de 1905, la mention de l'entité l'emporte presque toujours sur le nom de son directeur. Et déjà en 1915 pour le comité Vayreda, puis en 1917 pour le comité Battle et le comité Maragall, les appels à souscription n'indiquent que le nom des associations. L'effet de masse est saisissant.

De 1860 à 1930, les archives municipales gardent les traces de quatre-vingt-huit pétitions concernant les noms de rue. Globalement, le rythme pétitionnaire augmente et reflète une véritable prise de parole. Les pétitionnaires changent aussi: au début, ce sont des personnalités ou plusieurs propriétaires arguant de leur qualité. En 1895 apparait la première association de propriétaires regroupant les habitants de six rues de la Barceloneta. L'ampleur de la mobilisation est tout autre: en 1907, une pétition de la rue Burriana rassemble vingt-six habitants. En 1909, celle de la rue Fasthenrath en réunit cent six et celle de la rue Claveguera cent soixante et onze! Mais la mobilisation décroît dans les années Vingt. Le regroupement d'individus fait long feu. 
Les unions d'associations l'emportent alors. La première, en 1910, rassemble deux associations en faveur du nom de Lequerica, député aux Cortes de Cadix en 1812. La plus importante pétition reçue réunit vingt-cinq associations (soit 1752 signatures) en faveur du nom de Fabra y Puig, un industriel du textile. De la pétition individuelle au rassemblement d'entités prestigieuses, l'organisation sociale de la pétition s'est amplifiée et complexifiée.

Les pétitions peuvent mettre en œuvre des stratégies de persuasion: la plus performante consiste à obtenir l'appui du Teniente de Alcaldia qui dirige l'arrondissement et qui se fera l'avocat auprès du conseil municipal. Une autre stratégie consiste à attendre les meilleures conditions politiques pour voir triompher sa demande: en novembre 1923, les habitants de l'ancienne rue Santa Madrona expriment leur joie de voir instaurée la dictature; ils demandent la suppression du nom du général Moragas, héros du catalanisme, expliquant qu'ils ne manifestèrent jamais aucune plainte, "persuadés de l'inutilité de raisonner sous le régime précédent ". En cas d'échec, la pétition cherche à gagner à sa cause des " poids lourds » de la vie sociale barcelonaise: les habitants de la rue Claveguera (" égout ", en catalan), déboutés en 1921, réitèrent une pétition par l'intermédiaire de la puissante Cámara Oficial de la Propiedad Urbana.

La stratégie du nombre peut être évaluée à ses résultats: on sait d'abord que la pétition ne représente que $15,5 \%$ des initiatives en matière de noms de rue. Promotrice de mémoire de second rang, la pétition est néanmoins efficace: $80 \%$ de réponses favorables entre 1860 et $1898,73 \%$ entre 1907 et 1923 , et seulement $57 \%$ sous la dictature. Même si elle est un moyen de pression qui perd en efficacité, la pétition est payante.

\section{La souscription n'est pas populaire}

La popularité se mesure également à l'étendue et la nature des souscriptions. L'histoire de la souscription distingue nettement deux périodes: avant 1896, les donateurs affichent un geste de charité sociale qui s'estime à l'importance du don. Avec l'émergence du catalanisme, la souscription devient populaire et massive.

Au départ, la souscription est un geste de charité. L'objectif d'une souscription levée en 1860 est [d'] « ériger un monument en commémoration de la guerre d'Afrique, et [de] créer un fond de pension pour les familles des blessés de guerre ». En novembre 1869 un autre projet de monument aux combattants du Pont d'Alcolea prévoit d'inscrire sur la colonne les noms des donateurs, comme rétribution symbolique de leur contribution économique. Le même principe est avancé pour le monument Rius i Taulet et pour le mausolée aux soldats des guerres de Cuba et des Philippines. C'est pourquoi la cérémonie du XIXe siècle s'accompagne souvent de distributions de «bons aux pauvres »: lors de l'inauguration de la statue de Prim en 1887 par exemple, la municipalité distribue mille ptas en bons aux Volontaires des guerres d'Afrique.

Si le caritatif se confond avec le commémoratif, c'est que les deux activités sont com- 
plémentaires au regard des experts comme à celui des classes dominantes. Le devoir de charité et le devoir de mémoire expriment un même sentiment de dette contractée envers les soldats ayant consenti des sacrifices pour satisfaire les rêves de grandeur des dirigeants. La charité et le souvenir sont la rançon d'une position sociale élevée dans une société strictement hiérarchisée: noblesse oblige. En somme, cette intimité de départ exprime parfaitement la réalité de la commémoration comme activité élitaire.

L'analyse des listes de souscripteurs montre que la mémoire est l'affaire des élites. En effet, la souscription de la fin du XIXe siècle est insuffisante pour financer la totalité des projets. Les commissions exécutives finissent toujours par requérir l'aide de la municipalité pour combler le déficit qu'elles ont creusé par trop d'ambition. En fait, la nature élitiste de la souscription limite forcément l'assiette de souscription à quelques personnalités ou à quelques entités déterminées. Tel article de El Diluvio se moque du caractère prétendument populaire de la souscription à Rius i Taulet: «La souscription populaire s'est réduite, comme chacun sait, à quelques dons octroyés par un nombre infime de collectivités et d'individus et à une mauvaise plaisanterie, plutôt coûteuse, que ces messieurs de la Commission exécutive se sont permis d'infliger à notre budget municipal $»^{2}$.

À la fin du XIXe siècle, les souscripteurs sont donc peu nombreux. Par exemple, le buste d'Aribau est financé par deux souscriptions: la première, en 1877, rapporte 4690 ptas. La seconde, ouverte en décembre 1884, rapporte 1843 ptas réparties seulement entre dix-neuf personnes, soit une moyenne de 97 ptas chacune: des sommes énormes! De même, les 47000 ptas de la souscription au monument Rius y Taulet, de décembre 1899 à février 1902, proviennent de cinq cent soixante-dix participants, soit un don moyen de 82,5 ptas. Au XX $X^{\mathrm{e}}$ siècle, très peu nombreux sont les monuments qui recourent encore à ce modèle de souscription.

\section{Naissance de la souscription populaire: les cas Güell et Clavé}

Les Commissions exécutives cherchent à élargir le champ des souscripteurs à cause des difficultés financières. La commission du monument Güell est la première à le réaliser à grande échelle: entre mars 1878 et janvier 1882, la souscription (dont nous ignorons le résultat final) recueille les signatures de 16611 particuliers, quinze municipalités, deux banques, vingt-huit sociétés industrielles et agricoles et cinq Députations provinciales, équivalent espagnol du département. On a bien affaire à une souscription d'un genre nouveau.

2. El Diluvio, 28/09/1901. «Lo de la suscripción popular (...) se redujo como es público y notorio a unos cuantos donativos debidos a un reducido número de colectividades e individualidades y a un bromazo asaz costazo y pesado que los señores de la Comisión Ejecutivo se permitieron dar a nuestro erario municipal ». Souligné dans le texte. 
Un extrait des listes, publié dans El Diario de Barcelona, consigne 1690 noms, soit environ $13 \%$ du total des souscripteurs. L'échantillon contient trois types de souscripteurs: 158 individus riches et patrons importants, des collectifs d'entreprise (dans ce cas, la liste précise les noms et dons de chaque employé) et des associations et entités diverses (Casino, Cercle catholique, etc.). Ce cas est très original car la majorité des dons provient surtout des entreprises industrielles (cristal, chimie, textile) petites et moyennes. Trois géants de l'industrie sont néanmoins présents avec 339 ouvriers (Fábrica de Cristal), 151 ouvriers (Fábrica Soler y Biosca) et 103 ouvriers (Fábrica Carlos Le Boeuf). Le montant des dons reflète la hiérarchie interne de l'entreprise: 50 réaux et plus pour les directeurs en tête de liste, entre 5 et 20 pour les chefs, moins de 2 réaux pour les employés. La moyenne des contributions est très faible, entre 0,36 ptas et 0,18 ptas par employé. Le caractère populaire de la souscription est ici indéniable.

Avec 16000 signatures, la souscription Güell appartient déjà à la famille des grandes souscriptions de masse du siècle suivant. L'initiative appartient en priorité à des chefs d'industrie qui ouvrent dans leurs établissements des livres de dons. Dans ces conditions, une certaine contrainte s'instaure et la souscription peut s'apparenter à une contribution obligatoire pour l'ouvrier, un impôt idéologique. Il ne faut pas non plus exclure une participation volontaire des employés sensibilisés par les grandes campagnes protectionnistes relayées par la presse populaire. En tout cas, la souscription se révèle un formidable instrument de mobilisation politique qui participe de l'affirmation d'une opinion publique. La souscription Güell présente toutes les caractéristiques d'un cas de transition.

La souscription relative au monument Clavé présente un caractère nettement plus moderne car l'initiative appartient en effet à un groupement d'associations chorales. La collecte est le produit de dons effectués pendant des concerts. Cette expérience originale connaît également des problèmes de trésorerie ${ }^{3}$ : reposant uniquement sur la générosité d'un public authentiquement populaire, la quête accuse les conséquences de la crise de 1882 et des épidémies de fièvre jaune qui s'ensuivent. Comme l'explique l'un des rapporteurs de la Commission, Conrad Roure:

La commission ne put développer toute son activité à cause de la crise économique que traverse le pays. (...) Lexiguïté des fonds qu'on a recueillis ne permet pas de donner aux travaux cette impulsion qu'ils auraient connue en un temps de prospérité économique et d'état sanitaire normal.

Quoi qu'il en soit, ce modèle de souscription privé reposant sur l'unique initiative du tissu associatif demeure exceptionnel dans les années Quatre-vingt. En cela, la souscription Clavé annonce l'avenir.

3. En décembre 1888, la Commisson implore la Députation et la municipalité de combler le déficit: "Malgré tous les efforts de cette commission pour combler le déficit qui nous reste, il manque encore la quantité de 7000 ptas ». 


\section{L'âge des souscriptions de masse}

Au tout début du XXe siècle, les monuments catalanistes au Dr Robert et au poète Verdaguer inaugurent une nouvelle étape de l'histoire de la souscription. On connaît bien le détail de trois souscriptions importantes: celles du Dr Robert (1902), celles de Verdaguer (1902-1903 mais surtout 1913-1914), et celle de Guimerà pour un monument jamais réalisé (1924-1925). À ceci s'ajoute des informations partielles sur les souscriptions aux monuments catalanistes Rius y Taulet (1900), Maragall (1913), et aux deux monuments républicains, Pi y Margall (1917) et Ignasi Iglesies (1928).

Les sommes recueillies sont importantes: 96500 ptas pour le monument Rius, 120268 ptas pour le docteur Robert, 139857 ptas pour Verdaguer, 250127 ptas pour Pi y Margall et seulement 59283 ptas pour Guimerà. En fait, les souscriptions sont comparables car les autorités (municipalité, Députation et éventuellement l'État) y participent en moyenne pour plus de la moitié, excepté pour la dernière: la collecte populaire avoisine donc toujours les 60000 ptas.

Le rythme des collectes est comparable dans trois cas sur quatre. Le coup d'envoi très rapide assure entre 60 et $70 \%$ des dons en deux ou trois mois; puis, le rythme décélère nettement. Dans le cas Guimerà seulement, la souscription connaît une augmentation très régulière, sans doute imputable au mode de diffusion d'une commémoration de résistance, en pleine dictature militaire. Dans la mesure où tout se joue généralement dans les premières semaines après l'ouverture des listes, leur prolongation est improductive: on mesure la popularité et le succès de la collecte à la vitesse à laquelle la souscription est couverte. Pour le Dr Robert, quatre mois et demi suffisent à réunir 120000 ptas. Pour Guimerà, huit mois sont nécessaires. Mais pour Verdaguer, il faut compter dix-neuf mois de souscription. Celle de Rius y Taulet, la plus lente de toute, met vingt-cinq mois à réunir 47000 ptas!

L'élargissement du bassin de souscription, déjà perceptible à la fin du XIX ${ }^{e}$ siècle, est considérable. Mais peut-on connaitre le nombre approximatif de donateurs? C'est difficile car les listes de souscription précisent rarement leur nombre: les associations, les municipalités, les entreprises envoient des sommes globales sans spécifier ni le nombre ni les noms de leurs donateurs. Pour le monument Guimerà, on a 8424 personnes pour la moitié de la somme totale: par extrapolation, le nombre total de souscripteur serait donc de 17000 personnes environ. Pour le monument Iglesies en septembre 1928, on aurait 8000 signatures selon La Publicitat. C'est sans compter les nombreux dédits: entre juin 1903 et mai 1904, la Commission exécutive du monument à Pi y Margall enregistre 66029 ptas de dons. Mais à l'ouverture de la seconde souscription en 1912, les caisses n'en contiennent que 2846 ! On découvre que même la municipalité de Barcelone a fait défaut à ses promesses de participation!

Il est par conséquent difficile d'établir des données fiables: on peut estimer qu'au début du $\mathrm{XX}^{\mathrm{e}}$ siècle, une souscription populaire rassemble entre 15000 et 
20000 signataires, probablement plus. La souscription est désormais un phénomène de masse.

\section{Portrait-robot du donateur}

Avec la massification, le profil sociologique du donateur change.

Tout d'abord le souscripteur est généralement un homme. Les noms féminins relevés sur les listes de la fin du XIXe siècle sont ceux de veuves ou bien d'épouses, accolés à celui du chef de famille. Lorsque le sexe du donateur est précisé, on note une participation féminine minoritaire mais croissante, jusqu’à $8,3 \%$ pour la souscription Guimerà (1924). Dans ce cas, on compte également cinquante-deux enfants, preuve supplémentaire de l'approfondissement des cercles de collecte. Globalement cependant, le geste souscripteur s'apparente au vote: il est réservé au chef de famille, adulte et masculin. 15000 à 20000 souscripteurs représentent environ $15 \%$ du corps électoral barcelonais au début du siècle. Ce qui équivaut en 1901, à la veille de l'élan nationaliste, au nombre de votants.

Ensuite, le souscripteur semble majoritairement barcelonais. En 1912, pour la souscription Verdaguer, seulement $8 \%$ des associations participantes sont catalanes et nonbarcelonaises. C'est encore bien peu. En 1924 pour la souscription Guimerà, la participation provinciale représente un quart des contributions personnelles, lesquelles contribuent pour $52,3 \%$ des sommes souscrites. Ces indices confirment donc la percée tardive mais réelle de la province catalane entre 1912 et 1924 qui s'identifie de plus en plus évidemment avec la mémoire de la capitale catalane. Le nombre croissant de délégations provinciales lors des cérémonies le confirme par ailleurs.

Enfin, la souscription de masse mobilise en priorité dans les riches couches intermédiaires urbaines. Pour le monument Verdaguer (1912), le poids économique des associations économiques et professionnelles (31,2\% et $10 \%$ respectivement) indique clairement la place occupée par les classes supérieures: les patrons, médecins, avocats, pharmaciens, notaires, juges, etc. sont nombreux. La souscription Guimerà (1924), la plus précise de toutes, indique une très forte proportion d'employés issus d'entreprises petites et moyennes du commerce et de la boutique ("Dépendants », $52 \%$ ). Ensuite, viennent les " groupes d'ouvriers et d'employés » $(15 \%)$, les employés de banque $(9 \%)$ et les fonctionnaires (3\%). Il reste $21 \%$ d'ouvriers (" obrers ", " operaris ») de formations diverses: textile et blanchissage, construction électrique, ferroviaire et automobile, porcelaine et taille de pierre, métiers du livre et de l'impression. Les nouvelles couches intermédiaires nées de la puissante révolution industrielle catalane sont là.

La petite bourgeoisie des employés et des patrons occupe donc la première place parmi les souscripteurs; la classe ouvrière montre des signes d'intérêt notable qui prouvent son intégration à un monde de la commémoration en voie de démocratisation, bien que globalement, il faille attendre le début des années 1920 et surtout la IIe République 
pour les voir s'y intéresser. Au total, la composition de la souscription reflète la structure sociale dominante: au modèle élitiste fondé sur une aristocratie de l'argent se substitue un modèle de masse drainant les classes moyennes. Le principe participatif l'emporte donc sur le principe représentatif.

\section{L'association, moteur de la participation}

La massification de la participation met en relief l'importance du cadre associatif dans l'encadrement des foules commémorantes.

Entre le monument Colomb (1885-1888) et le monument Verdaguer (1912), la catégorie regroupant les individus souscrivant à titre personnel s'effondre (de 24 à 4,9\%). Cela signifie entre autre chose que l'individu préfere s'intégrer et s'afficher dans un groupe: inversement, les associations qui ne représentaient que $1,7 \%$ des dons vers 1885 élèvent leur participation à $27 \%$ en 1912, sans compter les listes dressées par municipalités $(1,4 \%)$. En vingt ans, l'association s'est imposée comme cadre naturel de la souscription.

Qui sont les associations concernées? En nombre comme en contribution, les associations culturelles et politiques ont une place centrale qui en font le moteur de la souscription. Mais la contribution des associations de défense des intérêts économiques qui réunit les classes propriétaires montre le poids encore considérable occupé par les structures associatives élitaires. À défaut de pouvoir dresser avec exactitude le détail des associations souscriptrices, on peut analyser les listes d'associations présentes lors des cérémonies d'inauguration.

La mobilisation associative est très inégale. Un premier ensemble regroupe des manifestations intimistes où le nombre d'associations ne dépasse jamais la dizaine. Les cérémonies du Parc de la Citadelle en sont le modèle: sept entités présentes pour le buste d'Aguiló, sept pour celui de Lleó Fontova, dix pour celui de Pepita Texidor. Un deuxième groupe, réduit, concerne les cérémonies de masse: 86 entités pour la pose de la première pierre du monument Robert (1910), 198 pour celle du monument Verdaguer (1914), 109 pour celle du monument Pi y Margall (1915). Un groupe intermédiaire réunit les cérémonies les plus nombreuses: trente-quatre entités pour le monument Pitarra (1906), 39 pour une plaque en l'honneur de Maragall (1917), vingt et un pour la plaque de rue à Martí i Julià (1921), etc.

Au-delà de cette typologie dont les limites explicatives sont évidentes, la question est de savoir quel type d'association préside la commémoration. Seule l'exceptionnelle longévité de la commémoration du Onze Septembre, la fête nationale catalane, de 1906 à 1922 permet de dresser des statistiques concluantes.

Première constatation, comme pour les souscriptions, l'association est reine, sans que 
la présence d'offrandes déposées à titre personnel, à partir de 1911, ne change en proportion. Si le nombre d'hommages isolés croît parallèlement à celui des hommages collectifs, il ne dépasse jamais la proportion de $20 \%$ des couronnes: l'association, quelle qu'elle soit, est bien le cadre incontesté de la commémoration. De plus, la proportion d'associations politiques va décroissant (de $80 \%$ en 1911 à $52 \%$ en 1922), la part de la presse et des associations professionnelles étant négligeable. À l'inverse, l'importance des entités culturelles croit régulièrement jusqu'en 1919 (de 15,3\% en 1912 à 29,1\% en 1919) puis reflue légèrement $(19,8 \%$ en 1921 et $16,5 \%$ en 1922) à cause de la forte poussée des délégations municipales et étrangères après la première guerre mondiale, résultat des conquêtes électorales des années Dix. Parmi les entités culturelles, le poids des groupes choraux et autres orphéons est remarquable (entre 5 et $6 \%$ ), et celui, plus irrégulier, des institutions d'enseignement (autour de $4 \%$ ). À partir de 1913, excursionnistes et sardanistes occupent une place secondaire (entre 2 et $3 \%$ ). Le restant regroupe des cercles artistiques, des groupes dramatiques, et pour la première fois en 1916, des associations sportives (pour ces dernières, entre 1 et 1,5\% de 1919 à 1922). La trilogie des associations chorales, sardanistes et excursionnistes est à la base de la mobilisation nationaliste.

Souscription et participation aux cérémonies confirment le rôle moteur des associations culturelles et politiques dans la mobilisation collective. Globalement, les associations culturelles sont plus nombreuses dans la souscription, les entités politiques plus présentes lors des cérémonies, sans que l'on sache si cette répartition correspond à deux modes de mobilisation différents mais complémentaires. Si les rôles sont peut-être différenciés, les associations culturelles et politiques sont bien les premières cellules sociales de mémoire. La primauté du nombre ne s'accompagne donc pas d'une atomisation de la société souscrivante.

\section{Un noyau dur commémorant}

Existe-t-il des groupes moteurs susceptibles de cristalliser une présence associative massive?

L'analyse des cérémonies du Onze Septembre permet de calculer le taux de renouvellement du tissu associatif sur une série continue de 1911 à 1919. Le pourcentage d'associations ayant déjà participé à la cérémonie une fois précédente (mais pas nécessairement l'année immédiatement antérieure) est relativement stable: il varie entre $60 \%$ (1912) et $88,3 \%$ en 1917 et se fixe en moyenne autour de $72 \%$. En moyenne donc, trois associations sur quatre ont déjà assisté à la cérémonie.

En étudiant attentivement la composition des délégations, on peut déterminer un noyau dur d'associations militantes, présentes invariablement chaque année. L'envol des chiffres de participation (300 associations en 1923, 296 en 1922) modifie certes leur poids relatif; mais en tant qu'entités vétérantes, elles sont un plus symbolique 
important qui donne à leur présence un relief particulier. En 1916, ces associations forment la Junta Permanent chargée chaque année d'orchestrer la commémoration sous la direction tournante de l'une d'entre elles.

D'autres associations forment un second cercle de fidèles, puis un troisième, etc. Cette structure «en pelure d'oignon » est tout à fait caractéristique du nationalisme catalan; les nationalistes fervents ne sont-ils pas d'ailleurs appelés " ceballuts " (l'oignon en catalan)? Le cœur associatif dense est formé d'une vingtaine de groupes que l'on retrouve sur le devant de la scène lors des moments difficiles, comme en 1917. Dans ce noyau agglutinant, les associations sont très diverses: un groupe choral peut avoir la même valeur symbolique qu'un parti ou un club d'excursionnistes. Autour de ce noyau s'ajustent d'autres séries d'entités qui amortissent éventuellement les coups portés au monde nationaliste pendant les périodes de répressions et les dictatures car elles disparaissent en premier. Par lente sédimentation, les associations les plus ferventes gagnent en prestige tandis que les plus récentes venues, plus volatiles sans doute, attirent de nouvelles associations en nombre exponentiel, comme en atteste le nombre de couronnes déposées au pied du monument: de 40 en 1907 à 377 en 1920.

En fin de compte, la cebolla nationaliste fait preuve d'une remarquable stabilité malgré son expansion formidable. Les interactions sociales développées lors des cérémonies s'organisent selon des réseaux relativement invariants. La commémoration permet l'activation de ces relations et leur approfondissement et consolide la communauté nationaliste au-delà des aléas de la vie politique. Mais pour grandir, la communauté doit développer des stratégies de mobilisation.

\section{"Als Catalans!"}

L'appel des commissions monumentales à l'opinion publique est primordial. Lorsque les promoteurs de mémoire en appellent au public, ils reconnaissent implicitement l'existence d'une instance critique et son droit de regard. Mais la reconnaissance de l'opinion publique est longue à s'instaurer.

Trois opinions au cours du temps reflètent l'évolution de l'attitude des promoteurs de mémoire. Première opinion: en 1855, après que la municipalité décide d’orner la place Royale d'une statue équestre dédiée à Ferdinand le Catholique, un conseiller se félicite « de l'éloge qu'ont fait certains journaux de la Cour des mérites de la statue ». Ici, le tribunal de l'opinion qui exprime la mesure du bon goût est à Madrid. La parole autorisée, celle qui compte et qui est prise en compte par les promoteurs est extérieure à la Catalogne. Cela ne signifie pas que les Barcelonais n'aient pas d'avis sur la question mais simplement que les promoteurs ne s'en soucient pas.

Deuxième opinion: en 1882, le secrétaire de la Commission exécutive du monument à Colomb, Carles Pirozzini, défend la prééminence du jury pour le choix du sculpteur: "Ces Messieurs du Jury n'ont absolument pas besoin de l'opinion publique pour la 
connaissance parfaite des œuvres qui sont soumises à son examen et à son jugement ". Ici, Pirozzini est parfaitement conscient de l'affirmation d'une opinion publique autonome et critique; simplement, il en récuse la compétence et refuse de la prendre en compte. Il s'agit de défendre la parole de l'expert contre celle du quidam, d'affirmer la légitimité d'une opinion contrôlée contre celle de la rue. Ce faisant, Pirozzini admet déjà l'éventualité de la critique et la difficulté quil y a à la contourner sans compromettre les chances du culte.

Troisième et dernière opinion: en 1928, alors qu’on s'apprête à célébrer l'anniversaire de la conquête de Majorque par Jaume Ier en 1229, le président de la commission d'hommage avoue: "Commemorar, és opinar "4. Commémorer, c'est avoir et donner son opinion: il n'est plus question d'échapper à l'opinion, encore moins de l'exclure; l'expertise est désormais l'un des attributs de l'opinion publique.

Ces trois attitudes illustrent comment une opinion publique est progressivement érigée en tribunal de mémoire. On sait bien l'intérêt qu'il y a à invoquer une opinion publique unanime: il s'agit de légitimer le promoteur qui s'en réclame et de renforcer dans les conflits de mémoire les rapports de force qui fondent sa position. Mais l'opinion publique est loin de constituer une donnée historique a priori. Sa naissance, sa constitution et l'invention de ses modes d'expression sont liées à l'histoire de prises de parole critiques dans la société. Les promoteurs feignent de l'ignorer, acceptant de se soumettre à ce qu'ils croient constituer un jugement infaillible et populaire. La presse joue alors un rôle fondamental de formation, d'expression, de contrôle et de conquête de l'opinion publique ainsi pensée.

\section{Moyens de conquête de l'opinion publique}

Le défi de la publicité exige la mise au point de méthodes de communication pour atteindre et mobiliser le public. C'est pourquoi les comités et commissions exécutives affectionnent particulièrement la présence d'un homme de presse en leur sein: le directeur du Noticiero universal pour le monument Clavé, celui du Diario de Barcelona pour les monuments Aribau et Rius i Taulet par exemple. Un cas exemplaire est celui de Francesc Matheu i Fornells, promoteur des bustes du Parc de la Citadelle consacrés aux personnalités de la Renaixença, la renaissance culturelle à partir de 1840 .

Mais dans la majorité des cas, la presse se limite à un rôle de caisse de résonance et non d'initiatrice. Pour preuve, le rôle ambigu de la presse lors d'une souscription où les quotidiens, sans avoir l'initiative de la collecte, se chargent de publier les noms des donateurs et les montants des dons. Cette publicité constitue la rétribution symbolique du don sous forme de prestige social. Non seulement la presse offre les moyens du succès de la souscription mais constitue en fait l'une de ses fins.

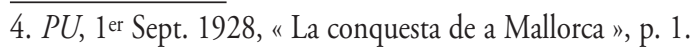


Pour la souscription au monument du Dr Robert par exemple (1901), La Veu de Catalunya commence à publier les listes de donateurs avant même que ne se forme une commission exécutive. 16000 ptas sont réunies dès le jour du décès du patricien, le 17 avril 1901. Puis, La Vanguardia et El Diario de Barcelona le 24, La Renaixença le 30 ouvrent leurs colonnes à la souscription. L'engouement est si rapide que le suivi de la souscription est quotidien: l'ascension vertigineuse des sommes crée et entretient la souscription par effet d'entraînement. Sur les 120268 ptas que reçoit la Commission exécutive, $95 \%$ ont transité par la presse et $83,7 \%$ uniquement dans La Veu de Catalunya, le principal organe du camp catalaniste.

Ces faits permettent de mesurer la puissance de l'effet publicitaire. Et les promoteurs de mémoire ne s'y trompent pas: en novembre 1912, le président du comité Verdaguer visite chaque directeur de journal, et lui demande de "publier toute la propagande possible pour que la subvention récemment ouverte donne le meilleur résultat possible ". C'est dire qu'à chaque étape importante de la politique de mémoire, les commissions tâchent de faire la publicité des actes: appel à souscription, formation des comités et jurys, comptes rendus des concours artistiques et des travaux en cours, publication des dates et lieux de commémoration, des ordres de défilés, des recommandations ultimes, etc.

En janvier 1900 par exemple, le Comité Rius y Taulet proclame ses intentions dans sept quotidiens barcelonais. Du 15 au 26 juin 1904, La Publicidad publie quotidiennement l'ordre de convocation de la cérémonie d'inauguration d'une plaque commémorative sur la façade de la maison natale de Pi y Margall. La campagne de presse culmine souvent avec la sortie d'un numéro spécial consacré au personnage honoré. Lorsqu'il y a lieu, la publication des commentaires, polémiques, débats et caricatures sous une rubrique spéciale contribue à augmenter l'effet publicitaire.

Il serait inexact de limiter la publicité à la seule action de la presse. Au début du XXe siècle, les outils de propagande se multiplient: en mai 1909, le comité pour l'hommage des Martyrs de l'Indépendance de 1809 prévoit que « seront fixés aux coins de rue et dans les lieux publics des allocutions enthousiastes incitant le peuple à concourir à la manifestation civique ». Pour le Festival Pi y Margall célébré en avril 1917, la commission organisatrice met en vente un album de quinze pages qui contient une présentation du futur monument et plusieurs articles de célébrités en faveur du projet. On vend aussi des cartes postales, des agrandissements photographiques de la maquette. Une loterie permet de gagner une médaille commémorative de l'événement. L'ingéniosité des promoteurs de mémoire ne s'arrête pas là: dès 1901, on peut acquérir une reproduction de la statue de Rafael Casanova, le héros de la fête nationale, de 1,20 mètre de hauteur pour 55 ptas seulement. Enfin, certains monuments se prêtent à la réclame, telle cette reproduction de Christophe Colomb tel qu'on le voit au sommet de son monument, mais tenant un Havane à la main! Trouvaille d'un commerçant du Pavillon des Colonies de l'Exposition Universelle de 1888. 


\section{La mobilisation associative}

À l'heure des souscriptions et des défilés, les associations sont de puissants vecteurs de mémoire auprès de leurs affiliés. On a déjà constaté leur efficacité pour collecter des fonds, pour l'exercice d'une forme de contrainte subtile sur tous les membres du groupe. Comment, en effet, échapper à l'obligation d'un don lorsque tous les adhérents d'une association s'y soumettent? Plus encore, il faut compter avec les innombrables conférences, réunions, hommages, veillées nécrologiques que les entités organisent dans leurs murs. Deux exemples suffisent à en montrer l'ampleur.

La semaine précédant la Diada Pi y Margall (« la Journée Py i Margall »), le dimanche 29 avril 1917, est l'occasion d'une mobilisation citoyenne hors du commun. Le journal El Poble Català s'en fait le fidèle écho: le 22, un hommage à Pi y Margall au Café Montero (place d'Espagne) puis une conférence à la Fraternitat republicana Instructiva "El Pueblo ", à El Clot, quartier ouvrier de la capitale. Le 24, réunion publique de la commission organisatrice, conférence de Roca i Roca, le chef du parti fédéraliste, suivi d'une tertulia au Foment del Trabajo Nacional. Le 25, trois hommages prévus aux sièges du Centre Nationalista Català, de l'Unió Gremial et à la Fraternitat del Clot. Le 27, une conférence au CADCI est précédée d'une soirée au Comité Républicain Fédéral de Barcelone. Le 28, un grand meeting est organisé par la Joventut Nacionalista Republicana. En sept jours, dix actes annoncent la Diada.

La commémoration du Onze Septembre est un autre modèle du genre. En plus de la traditionnelle conférence ou veillée nécrologique, s'ajoutent vers 1910 de nouvelles formes de rappel: le festival choral, le spectacle de sardanes et surtout le théâtre. Une soirée organisée le 11 septembre 1913 au Thêâtre Apolo, sur le Parallèle, programme quatre spectacles: « la représentation de l'épisode dramatique en un acte de Josep Burgas, Jordi Erin» dont l'action se déroule dans la jeune Irlande révoltée. Puis, « un grandiose tableau dramatique du même auteur, symbole du glorieux Corpus de Sang et intitulé: Els Segadors de Polonia", en référence à la guerre des Segadors de 1640 où la Catalogne se détacha momentanément de la Couronne d'Espagne. Suit le célèbre monologue de Mestre Olaguer, d'Angel Guimerà, qui a pour cadre dramatique le siège de 1714 que commémore le Onze Septembre. Enfin, «un tableau dramatique inédit en un acte de Manel Rocamora, Via Fora LLadres» (Dehors, voleurs!). Les entractes sont animés par une cobla (un groupe de sardane). Le prix d'entrée va de 0,3 à 1 pta.

$\mathrm{Au}$ total, les voies de la mobilisation populaire sont multiples. Elles attestent de la vivacité d'une véritable culture de la mobilisation qui suppose une stratégie de communication: effets d'annonce, effets de redondance à la veille d'une cérémonie... une gestion de l'information est indispensable, sans quoi la commémoration court au fiasco. C'est le cas de la cérémonie indépendantiste du Fossar de les Moreres, en septembre 1915, gâchée à cause d'ordres de convocation contradictoires. Lorsque le public parvient au Fossar, l'acte d'hommage est déjà terminé! 


\section{Le nombre, critère de légitimité de la mémoire}

Dans une société déjà démocratique, le nombre de commémorants est devenu la source unique de légitimité d'une entreprise de mémoire. Pour les promoteurs, l'assistance du public aux cérémonies, son enthousiasme à souscrire sont des épreuves de vérité en même temps qu'un jugement sur la validité de ses prétentions.

Pour discréditer une mémoire adverse et dénoncer son aspect artificiel, il suffit d'en évaluer le nombre de participants. Pour El Correo Catalán, le monument Pi y Margall est illégitime car « l'acte fut un échec retentissant; Barcelone n'assista ni adhéra à la fête. Les manifestants ne dépassèrent pas le nombre de 2000 ». À l'inverse, le nombre incalculable de participants est certainement la meilleure garantie de réussite avancée par les promoteurs de mémoire. La bataille des chiffres fait rage, comme si la capacité à rassembler et la légitimité d'une commémoration se mesuraient avant tout en valeur absolue. C'est pourquoi les articles regorgent de références au nombre d'assistants et s'efforcent d'apprécier le degré de participation des Barcelonais, tel La Renaixença du 13 septembre 1901 qui en évalue l'importance à la superficie de voie publique occupée:

Il nous est difficile de faire un calcul exact des milliers de personnes qui sont venues à la manifestation (...). Nous dirons simplement que la masse des manifestants (...) allait de la moitié du Salon de Sant Joan sur toute sa largeur, devant le palais de Justice, jusqu'au-delà de la rue Ali-Bey, alors que les larges carrefours des rues débouchant sur la promenade se trouvaient totalement encombrés.

Si le nombre est l'objet de tant de commentaires passionnés, c'est qu’il exprime symboliquement l'unanimité. Le nombre permet aux manifestants de s'ériger en représentants de la communauté entière: ce ne sont pas des centaines de Barcelonais qui sont rassemblés mais, métaphoriquement, tous les Barcelonais, le peuple. La Publicitat exprime parfaitement cette idée lors de l'enterrement de Ignasi Iglesias, en novembre 1928:

Il est impossible d'imaginer cet infini cortège. Dire un chiffre serait une chose bien aventureuse. Quand les foules atteignent ces proportions - des têtes et plus de têtes encore, par en bas, par en haut, on n'en voit jamais la fin-, il est impossible de compter: c'est tout un peuple, c'est le peuple, et le peuple est innombrable.

Une telle métonymie qui prend une partie de la société (les participants) pour le tout (la société catalane) repose en définitive sur la croyance que la mémoire est une manifestation spontanée et naturelle dans le corps social. Soit la cérémonie est en accord avec les souvenirs du peuple, et alors son succès et sa légitimité sont assurés; soit, elle est en désaccord et l'absence de la foule vient la sanctionner. La popularité serait donc la valeur 
de la commémoration en même temps que sa principale source de légitimité. Le nombre atteste finalement de l'authenticité de la mémoire.

En fin de compte, on est passé d'une société de mémoire élitaire où prédominait une personnalité " agglutinante » à une société de mémoire de masse où prédomine un noyau d'associations " agglutinant ». La structure fondamentale de la société commémorative, strictement organisée autour d'un noyau resserré, semble invariante. La logique représentative à l'œuvre au XIX $X^{\mathrm{e}}$ siècle ne disparât pas: le comité continue de réunir les meilleurs. Mais elle se complète par la dimension participative qui introduit un changement de nature de la commémoration. Le nombre importe plus que la qualité des participants. Quand le critère primordial de la commémoration devient sa popularité, elle est alors un signe manifeste d'une démocratisation de la société espagnole en Catalogne.

À l'âge des commémorations de masse, la société de mémoire tâche de conquérir une partie toujours plus importante de la société par l'usage de techniques de mobilisation de masse. Les promoteurs parviennent ainsi à faire passer leurs souvenirs particuliers pour des souvenirs collectifs. D'un côté, les promoteurs déploient de nombreuses stratégies de conquête de l'opinion. La nécessité de la publicité exige un savoir faire, un talent d'organisation qui est à l'opposé de la spontanéité. De l'autre côté, la foi du nombre, vérité ultime de la commémoration, repose sur la croyance d'une mémoire populaire naturelle qu'il suffirait de révéler et de canaliser, comme si le peuple était une entité réelle dotée de souvenirs et qu'il existait une mémoire collective sous-jacente aux efforts des promoteurs.

Le constat peut paraitre paradoxal: dans une société de masse, les politiques de mémoire s'efforcent de créer un large public nationaliste tout en postulant l'existence d'une mémoire collective sui generis. Si bien que la stratégie de conquête de l'opinion publique ne vaut que si la croyance est instituée dans la mémoire collective. Et lorsqu'une majorité de gens admet cette croyance, elle agit effectivement comme s'il existait réellement un peuple catalan singulier doté de souvenirs propres. D'une certaine manière, la mémoire collective acquiert peut-être un certain degré d'existence parce qu'au départ elle fut postulée et qu'on travailla en son nom. 